\title{
Beclin 1, an autophagy-related gene, augments apoptosis in U87 glioblastoma cells
}

\author{
XIN HUANG $^{1}$, QIANGQIAN QI ${ }^{2}$, XUMING HUA $^{3},{\text { XINYUAN } \mathrm{LI}^{3}, \text { WENCHUAN ZHANG }}^{3}$,

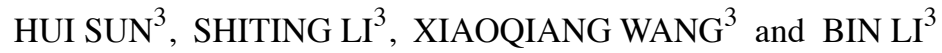 \\ ${ }^{1}$ Department of Neurosurgery, 171 Hospital, Jiujiang, Jiangxi 332000; ${ }^{2}$ Department of Neurosurgery, \\ Changzheng Hospital Affiliated to Second Military Medical University, Shanghai 200003; ${ }^{3}$ Department of Neurosurgery, \\ Xinhua Hospital Affiliated to Shanghai Jiaotong University School of Medicine, Shanghai 200092, P.R. China
}

Received November 30, 2013; Accepted January 21, 2014

DOI: $10.3892 /$ or.2014.3015

\begin{abstract}
Beclin 1 acts as a tumor suppressor and is an essential mediator of autophagy. Beclin 1 also interacts with Bcl-2 and can induce apoptosis by activating the mitochondrion permeabilizing function of proapoptotic multidomain proteins from the Bcl-2 family. Moreover, these Bcl-2 family members can activate autophagy by liberating Beclin 1 from its inhibition by Bcl-2/Bcl-XL at the level of the endoplasmic reticulum. We found that overexpression of Beclin 1 in U87 glioblastoma cells enhanced the capacity for cellular autophagy and induced apoptosis. Silencing of Beclin 1 decreased autophagic capacity but had little effect on apoptosis and cell proliferation. Beclin 1-Bcl-2 and Beclin 1-Bcl-xL complexes were detected by immunoprecipitation in cells that overexpressed Beclin 1. Furthermore, the levels of cytochrome $c$ in the cytosol and the activity of caspases-3/-9 in the cytosol increased after overexpression of Beclin 1. Our results suggest that Beclin 1 induces apoptosis via binding to $\mathrm{Bcl}-2$ and $\mathrm{Bcl}-\mathrm{xL}$, followed by the release of cytochrome $c$ into the cytosol and activation of caspases-3/-9.
\end{abstract}

\section{Introduction}

Autophagy is a conserved catabolic process that includes the sequestration of organelles and long-lived proteins residing in the cytoplasm into the autophagosome. Accompanied by a

Correspondence to: Dr Bin Li or Professor Shiting Li, Department of Neurosurgery, Xinhua Hospital Affiliated to Shanghai Jiaotong University School of Medicine, 1665 Kongjiang Road, Shanghai 200092, P.R. China

E-mail: libinmed@gmail.com

E-mail: 1sting66@163.com

Abbreviations: Atg, autophagy-related gene; Bcl-2, B-cell CLL/ lymphoma 2; Vps34, vacuolar protein sorting 34; hVps34, human Vps34; PI3k, class 3 phosphatidylinositol 3-kinase; LC3, microtubuleassociated protein 1 light chain 3

Key words: Beclin 1, LC3, Bcl-2, apoptosis, glioblastoma maturation process, the engulfed components are degraded by lysosomal hydrolases. Autophagic processes have been well characterized in yeast, and $>30$ autophagy-related genes have been identified in studies of yeast genetics (1). In addition to its role in the degradation of proteins and organelles, autophagy plays a critical role in cellular survival by providing energy during periods of metabolic stress. Nevertheless, depending on the cellular context and stimulus, autophagy may be indispensable for apoptosis by preceding and further turning on the apoptosis process [programmed cell death (PCD) type I] (2). Moreover, when stress conditions are excessive, autophagy becomes a cellular suicide pathway that works by digesting essential cellular proteins and structures. Autophagic cell death is also known as PCD type II (3).

As the mammalian ortholog of the yeast Atg6 gene, Beclin 1 is an essential mediator of autophagy (4-7). Beclin 1 forms a multimeric complex with vacuolar protein sorting 34 (Vps34) and class 3 phosphatidylinositol 3-kinase (PI3k), which is necessary for the formation of autophagosome. Accumulating evidence has shown that Beclin 1 plays a role as a tumor suppressor. Augmentation of Beclin 1 expression in MCF7 cells decreased their proliferation, clonogenicity in soft agar, and tumorigenicity in nude or severe combined immunodeficient mice $(4,8)$. Mice with heterozygous disruption of Beclin 1 suffer from a high incidence of spontaneous tumors $(5,9)$. Studies have also shown that the tumor-suppressing effects of Beclin 1 might also function by regulating autophagic cell death.

Members of the Bcl-2 family are important regulators of apoptosis (10) and include both anti- and pro-apoptotic members. Beclin 1, has been identified as a Bcl-2-interacting protein; this protein contains a $\mathrm{BH} 3$-like domain, which binds to the anti-apoptotic Bcl-2 family of proteins such as Bcl-2 and Bcl-xL, but Beclin 1 does not bind to the pro-apoptotic proteins Bax and Bak (11). The Bcl-2 protein modulates either apoptosis or autophagy through its interaction with Beclin 1 $(12,13)$. The levels of the Beclin 1 and Bcl-2 proteins in human pathogenic cells suggest the existence of an autophagy/apoptosis system dysfunction (14). Overexpression of Beclin 1 in MKN28 human gastric cancer cells augmented cis-diamminedichloroplatinum (CDDP)-induced apoptosis through an enhancement of the activity of caspase-3/-7/-9. These studies 
also suggest that the pro-apoptotic function of Beclin 1 may be through the inhibition of the anti-apoptotic function of Bcl-2 and Bcl-xL (8). Furthermore, a positive regulation of caspase-9 by Beclin 1 has been observed in HeLa cells. In this system, the autophagy-promoting activity of Beclin 1 was associated with the inhibition of cellular proliferation in the in vivo nude mouse model of tumorigenesis (15). Research examining the dietary bioflavonoid resveratrol (RV), demonstrated that $\mathrm{RV}$-induced apoptosis was prevented by RNA interference knockdown of Beclin 1 in human colorectal cancer cells. The authors conjectured that the autophagy and apoptosis pathways were strictly linked and merged at the execution point, with the caspases acting as death executioners downstream of autophagy (16). Beclin 1 may be the critical 'molecular switch' and may play an important role in regulating autophagy and apoptosis (15).

Malignant gliomas are the most frequent and lethal types of cancer originating in the central nervous system. Glioblastoma (GBM) accounts for $\sim 60-70 \%$ of malignant gliomas (17) and is the most biologically aggressive malignant glioma subtype. There has been marked progress in the understanding of the molecular pathogenesis of malignant gliomas $(17,18)$. A previous study demonstrated the low expression of Beclin 1 mRNA and protein in GBMs (19). We also confirmed Beclin 1 loss in GBMs using clinical specimens (20). However, the effect of Beclin 1 loss on the molecular genetics of the interaction between autophagy and apoptosis in GBM cells remains unclear. The present study explored the functioning of Beclin 1 in the induction of autophagy and the alteration of apoptosis-related proteins. Beclin 1 was both silenced and overexpressed in U87 GBM cells to determine if such major changes in the activity of Beclin 1 could revert the tumor phenotype of these cells.

\section{Materials and methods}

Cell lines and culture. Human GBM cell lines U87 and U251 were purchased from the American Type Culture Collection (ATCC; Manassas, VA, USA); these cell lines were grown and maintained in DMEM containing $10 \%$ fetal bovine serum (HyClone, Logan, UT, USA) on 2\% agarose-coated slides (Nunc, Roskilde, Denmark). Human astrocytes-cerebellar (HAc) astroglial cells were used as controls for these cells.

Real-time PCR. RNA extraction and RT-PCR analysis of the expression of Beclin 1 mRNA were performed as follows; cells were collected and total RNA was extracted with a spin column (Qiagen, Hilden, Germany) following the manufacturer's instructions. The first strand of cDNA was synthesized using a cDNA synthesis kit (Promega, Madison, WI, USA). The gene expression levels were analyzed by quantitative real-time PCR conducted on an ABI 7500 Real-Time PCR system (Applied Biosystems, Carlsbad, CA, USA). The primers used in the experiments were: forward, 5'-AGCTGGATGATGAGCTGAAGAG-3' and reverse, 5'-GATTGTGCCAAACTGTCCACTG-3'. After an initial incubation for $2 \mathrm{~min}$ at $50^{\circ} \mathrm{C}$, the cDNA was denatured at $95^{\circ} \mathrm{C}$ for $10 \mathrm{~min}$, followed by 40 cycles of PCR $\left(95^{\circ} \mathrm{C}, 15 \mathrm{sec}\right.$; $\left.60^{\circ} \mathrm{C}, 60 \mathrm{sec}\right)$. All results were obtained from at least three independent experiments. Using $\beta$-actin as an internal control, the mRNA levels of all genes were normalized.
Western blotting. Cellular protein preparations were prepared from the cell lines. Anti-Beclin 1 (Santa Cruz Biotechnology, Santa Cruz, CA, USA) and $\beta$-actin antibodies (Sigma, Milpitas, CA, USA) were incubated with the cellular protein preparations for $1 \mathrm{~h}$. The secondary antibody was added to these samples, and the resulting solutions were incubated for $30 \mathrm{~min}$ at room temperature in PBS-T containing 2\% BSA. Signals were detected by the ECL Plus Western Blotting Detection System according to the manufacturer's specifications (Amersham, Buckinghamshire, UK).

RNA interference. siRNA was designed using an siRNA Target Finder program (Ambion, Austin, TX, USA). The oligonucleotides that encoded the chosen siRNA sequences were designed for insertion into the pSUPER plasmid (OligoEngine, Seattle, WA, USA) following the published protocols (21). The candidate sequence-specific siRNA expression vectors were termed Beclin 1/siRNA1, Beclin 1/siRNA2, and Beclin 1/siRNA3; the negative control was abbreviated as pSUPER-non. Untransfected cells were used as controls. Stable transfection of pSUPER/Beclin 1 siRNA was performed in U87 cells using Lipofectamine 2000 (Invitrogen-Gibco, Carlsbad, CA, USA) following the guidelines provided by the manufacturer. The levels of knockdown of Beclin 1 mRNA and protein were determined by western blotting and real-time PCR analysis. We chose one of the siRNAs with the best inhibition efficiency for further study.

Evaluation of proliferation of GBM cells by the ${ }^{3} \mathrm{H}-\mathrm{TdR}$ incorporation test. Cells were incubated in 96-well microtiter plates. Then, $1 \mu \mathrm{Ci}$ of ${ }^{3} \mathrm{H}-\mathrm{TdR}$ (GE Healthcare, Fairfield, CT, USA) was added to each well and thoroughly mixed for $14 \mathrm{~h}$ before harvest. $\left[{ }^{3} \mathrm{H}\right]$ Thymidine incorporation was measured as counts per minute (cpm) as detected by a MicroBeta counter (Perkin-Elmer, Lincoln, RI, USA).

Assessment of U87 cell apoptosis by flow cytometry and Hoechst staining. Preparation of single U87 cells was performed as follows; apoptosis was induced in a $1 \times 10^{6}$ cells $/ \mathrm{ml}$ suspension by the addition of $1 \mathrm{mg} / \mathrm{ml}$ staurosporine. Cells were incubated in a $37^{\circ} \mathrm{C}, 5 \% \mathrm{CO}_{2}$ incubator for $1 \mathrm{~h}$. Next, $500 \mu \mathrm{l}$ of non-induced cell suspension was added to a second plastic $12 \times 75 \mathrm{~mm}$ test tube, followed by the addition of $5 \mu \mathrm{l}$ of Annexin V FITC conjugate and $10 \mu \mathrm{l}$ propidium iodide solution. The fluorescence of the cells was determined immediately (BD Biosciences, Franklin Lakes, NJ, USA). At the indicated times during treatment, cells were fixed on the dishes with methanol and stained for $10 \mathrm{~min}$ with Hoechst 33258 (Beyotime Institute of Biotechnology, Jiangsu, China) at $0.5 \mathrm{pg} / \mathrm{ml}$. The percentage of cells containing apoptotic nuclei was determined using fluorescence microscopy.

Immunofluorescence. U87 cells were incubated with a cyto C-specific (Epitomics) antibody. The cells were subsequently incubated with an FITC-conjugated anti-rabbit secondary antibody (Jackson ImmunoResearch Inc., West Grove, PA, USA). The mitochondria were counterstained with MitoTracker (Invitrogen, Carlsbad, CA, USA). Fluorescence microscopy was performed using a confocal microscope system (Leica Microsystems, Wetzlar, Germany). 


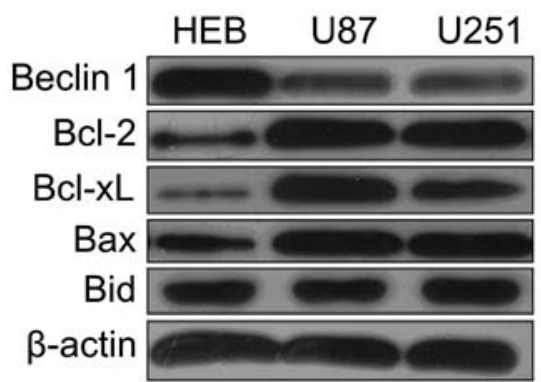

Figure 1. Expression of Beclin 1, Bcl-xL, Bax and Bid as detected by western blotting in U87, U251 and HEB cell lines. The expression of Beclin 1 was significantly lower in U87 cells. Bcl-2 family proteins were expressed at higher levels in $\mathrm{U} 87$ cells than in normal astrocytes. ${ }^{*} \mathrm{P}<0.001$ vs. control; ${ }^{* *} \mathrm{P}<0.001$ vs. low-grade. "Statistically significant changes. $\mathrm{P}<0.05$.

Co-immunoprecipitation. Transfected cells were lysed in cell lysis buffer $(50 \mathrm{mM}$ Tris- $\mathrm{HCl}, \mathrm{pH} 8.0,150 \mathrm{mM} \mathrm{NaCl}, 1 \mathrm{mM}$ EDTA, $1 \%$ Nonidet P-40, $10 \%$ glycerol with a mixture of protease inhibitors) for $1 \mathrm{~h}$. Whole cell extracts were collected and precleared. Beads coated with Beclin 1 antibodies were incubated with the precleared whole cell extracts at $4^{\circ} \mathrm{C}$ overnight. The beads were washed with cell lysis buffer four times. Finally, the beads were incubated in FLAG peptides or were boiled for $10 \mathrm{~min}$. The eluents were analyzed by western blotting to test for the levels of Bcl-2, Bcl-XL, Bax and Bid.

Statistical analysis. All data are presented as the means \pm standard error of the mean. For comparisons of the relative intensities of the western blot bands representing Beclin 1, Bcl-2, Bcl-xL, Bax, Bak, LC3, p62, cytochrome $c$ and caspase-3/-7/-8/-9 normalized to $\beta$-actin, an unpaired Student's t-test was used to compare differences between the paired transfectants (pcDNA3.1-Bec transfectants vs. PcDNA3 transfectants, pSUPER-Bec transfectants vs. pSUPER-non transfectants). The results were considered statistically significant when $\mathrm{P}$-values were $<0.05$.

\section{Results}

Expression of Beclin 1 and Bcl-2 family proteins in human glial cell lines. We initially examined the expression levels of Beclin 1 and Bcl-2 family proteins in U87, U251 and HEB cells (a human glial cell line) by western blotting. Next, we compared the expression levels of Beclin 1 in these cell lines. As shown in Fig. 1, the expression of Beclin 1 was significantly lower in U87 cells. Therefore, we chose the U87 cells as a model to investigate further the function of Beclin 1. In accordance with previous reports (22), the Bcl-2 family protein was expressed at higher levels in the U87 cells than in normal astrocytes (Fig. 1).

Autophagic capacity is regulated by Beclin 1 in U87 cells. To evaluate the function of Beclin 1 in autophagy, we altered Beclin 1 expression in U87 cells by introducing a Beclin 1 expression vector (pcDNA3.1-Bec) or an siRNA targeted to the Beclin 1 gene (pSUPER-Bec). As shown in Fig. 2, pcDNA3.1Bec transfectants showed greater Beclin 1 mRNA and protein expression than pcDNA3.1 transfectants (vector transfectants).
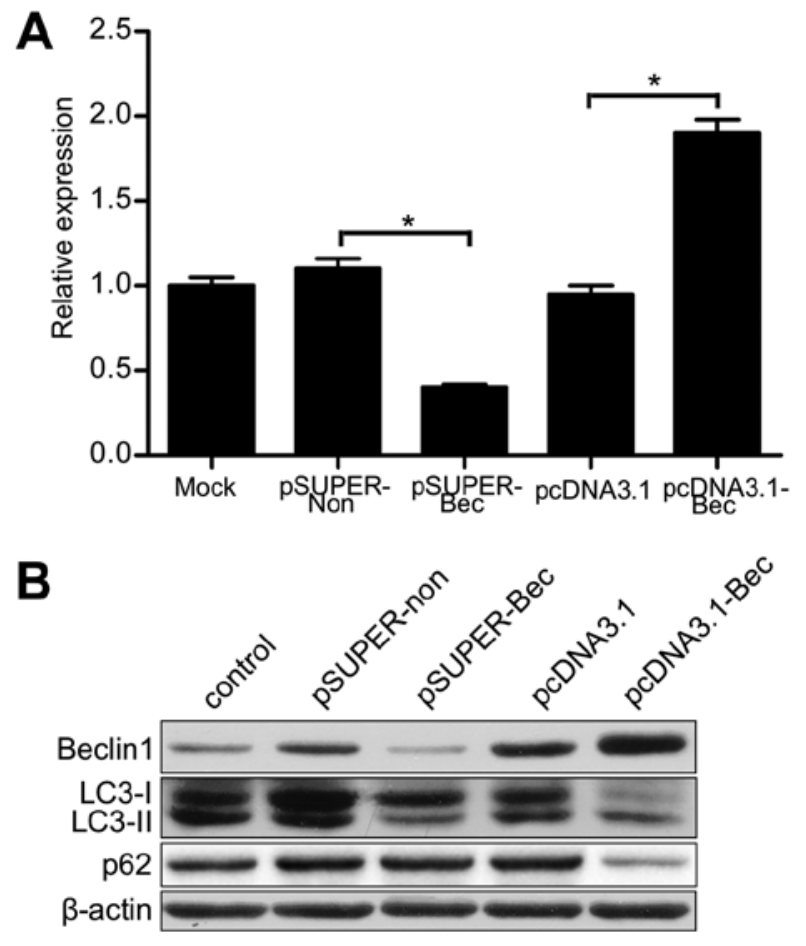

Figure 2. (A) Effect of transfection on Beclin 1 expression at $48 \mathrm{~h}$ using RT-PCR quantification. pcDNA3.1-Bec transfectants showed higher Beclin 1 mRNA transcription than pcDNA3.1 transfectants (vector transfectants). The mRNA of Beclin 1 was lower in pSUPER-Bec transfectants compared with pSUPER-non transfectants (scramble RNA transfectants). Bars, SD. ${ }^{*} \mathrm{P}<0.05$, statistically significant changes. (B) Protein expression of Beclin 1, LC3 and P62 in U87 cells transfected with Beclin 1/pSUPER-Bec compared with control cells. The results of the western blotting showed that the expression of LC3-II was increased in pcDNA3.1-Bec transfectants compared with vector transfectants or untreated cells, while LC3-II expression was markedly decreased in pSUPER-Bec transfectants. The level of p62 was lower in pcDNA3.1-Bec transfectants but higher in pSUPER-Bec transfectants than vector transfectants or scramble RNA transfectants.

Either the mRNA or the protein of Beclin 1 was significantly decreased in the pSUPER-Bec transfectants compared with the pSUPER-non transfectants (scramble RNA transfectants).

Autophagic capacity was monitored by LC3 and p62 evaluation by western blotting (23). The outcomes of western blotting showed that the expression of LC3-II was increased in pcDNA3.1-Bec transfectants compared with vector transfectants or untreated cells. However, LC3-II expression was markedly decreased in the pSUPER-Bec transfectants (Fig. 2B). Furthermore, the level of p62 was lower in pcDNA3.1Bec transfectants but higher in pSUPER-Bec transfectants than either the vector transfectants or the scramble RNA transfectants (Fig. 2B). These results indicated that the autophagic capacity was upregulated in pcDNA3.1-Bec transfectants but downregulated in pSUPER-Bec transfectants. These results indicate that Beclin 1 affects the capacity of autophagy in U87 cells.

Beclin 1 augments apoptosis and reduces cell proliferation in U87 cells. We then determined the effect of altered expression of Beclin 1 on the apoptosis of U87 cells. In pSUPER-Bec transfectants, there was no significant change in apoptosis compared with scramble RNA transfectants 
A

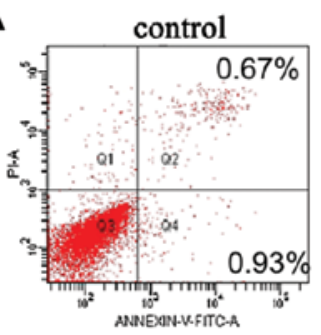

pSUPER-non

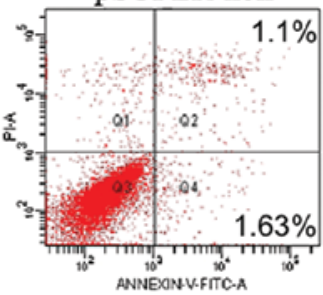

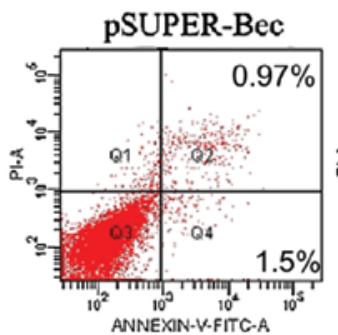
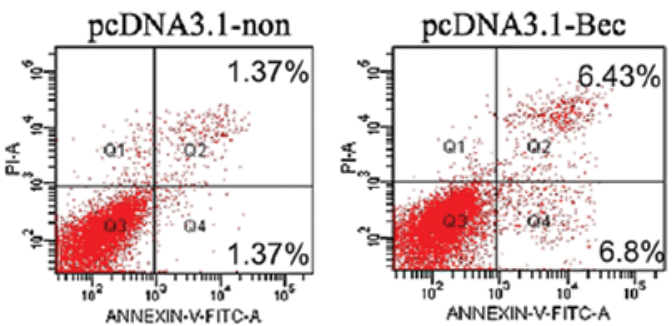

B

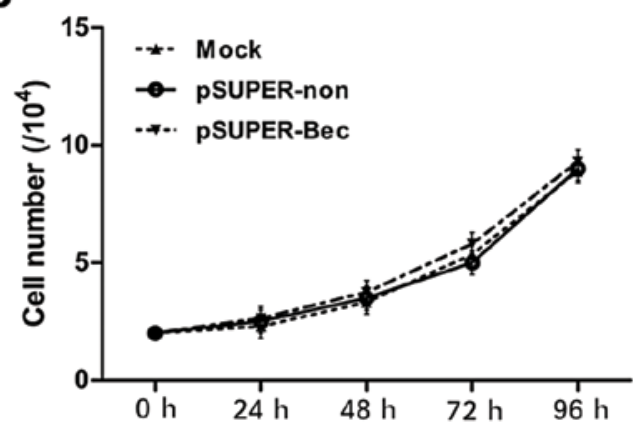

C

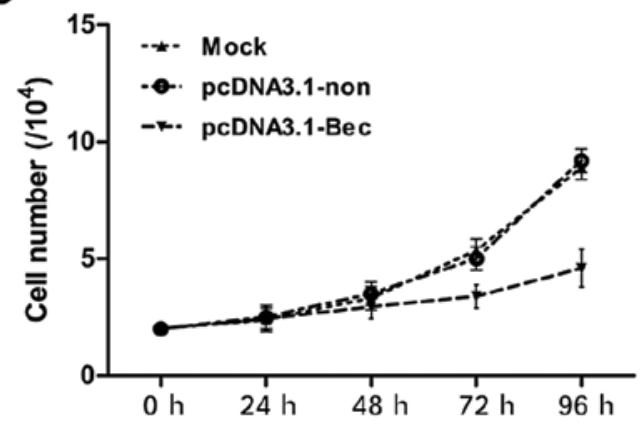

D

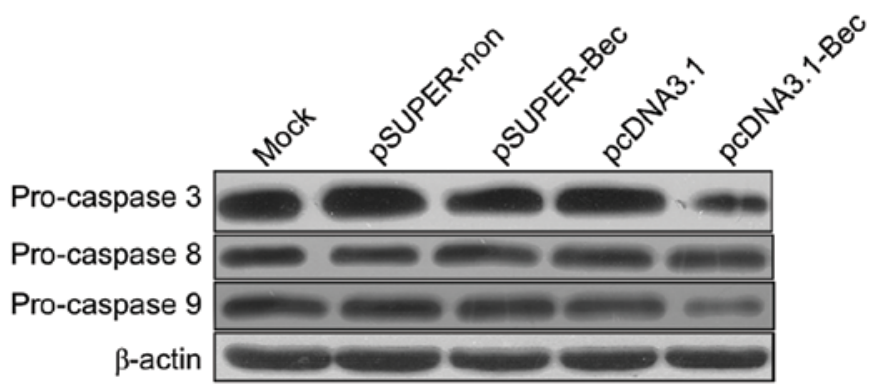

E

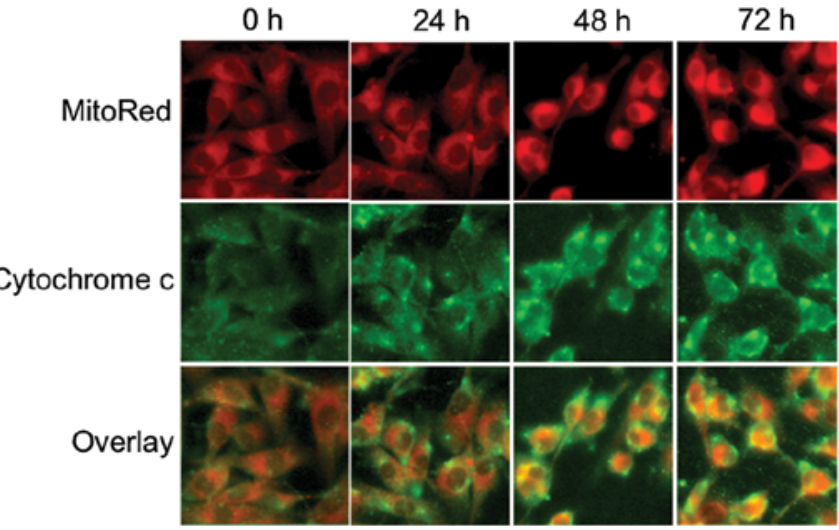

Figure 3. (A) Apoptosis, as measured by flow cytometry (FCM), of cells transfected for 48 h. In pSUPER-Bec transfectants, there was no significant change in apoptosis compared with scramble RNA transfectants. The rate of apoptosis was increased during the FCM assay in pcDNA3.1-Bec transfectants compared with other transfectants or untreated cells. (B,C) Overexpression of Beclin 1 inhibited cellular proliferation. The proliferation of U87 cells with each transfectant or with untreated cells was measured. Overexpression of Beclin 1 significantly suppressed the proliferation of U87 cells, whereas silencing Beclin 1 had no effect on cell proliferation. $\mathrm{P}<0.05$. (D) Immunodetection revealed pro-caspase-3, pro-caspase- 8 and pro-caspase- 9 expression in the cells following transfection for $48 \mathrm{~h}$. No significant difference in the increase of caspase- 8 activity was seen between the pcDNA3.1-Bec transfectants and the other transfectants. However, not only was there an increase in caspase-3/-7 activity, but there was also an increase in caspase-9 activity that was greater in pcDNA3.1-Bec transfectants than in other transfectants. (E) Immunofluorescence and immunoblotting detection of transfected pcDNA3.1-Bec. Cytochrome $c$ was released into the cytoplasm at a different time in the U87 cells. Cytochrome $c$ was visualized with FITC-conjugated anti-rabbit secondary antibody (green). Mitochondria were labeled with MitoTracker (red). Magnification, x1,000. The expression of cytochrome $c$ increased over time.

(Fig. 3A). Nonetheless, an obviously increased rate of apoptosis was observed in the flow cytometry assay in pcDNA3.1-Bec transfectants compared with other transfectants or untreated cells (Fig. 3A and Table I). The proliferation of each of the
U87 cell transfectants and the untreated cells was measured. Notably, overexpression of Beclin 1 significantly suppressed the proliferation of the U87 cells, whereas silencing Beclin 1 had no effect on cell proliferation (Fig. 3B and C). 
Table I. Apoptosis of transfected cells as revealed by flow cytometric sorting.

\begin{tabular}{lll}
\hline Group & $\begin{array}{c}\text { Q4 Proportion } \\
\text { of cells (\%) }\end{array}$ & $\begin{array}{c}\text { Q2 Proportion } \\
\text { of cells (\%) }\end{array}$ \\
\hline Control & $0.67 \pm 0.25$ & $0.93 \pm 0.25$ \\
pSUPER-non & $1.10 \pm 0.36$ & $1.63 \pm 0.40$ \\
pSUPER-Bec & $0.97 \pm 0.32$ & $1.50 \pm 0.60$ \\
pcDNA3.1-non & $1.37 \pm 0.55$ & $1.37 \pm 0.31$ \\
pcDNA3.1-Bec & $6.43 \pm 0.80^{\mathrm{a}, \mathrm{b}}$ & $6.80 \pm 1.05^{\mathrm{a}, \mathrm{b}}$ \\
\hline
\end{tabular}

${ }^{\mathrm{a}} \mathrm{P}<0.05$ vs. pcDNA3.1-non; ${ }^{\mathrm{b}} \mathrm{P}<0.05$ vs. control (one way ANOVA; $\mathrm{P}<0.05$, statistically significant changes).

Previous studies have reported that the formation of the Beclin 1-Bcl-2 or the Beclin 1-Bcl-xL complex may set Bax and Bak free, which could possibly trigger mitochondrial membrane disruption and the release of apoptogenic factors, such as cytochrome $c(24)$. We further analyzed the anatomical distribution of cytosolic cytochrome $c$ using western blotting immunofluorescence as previously described. This analysis revealed that cytosolic localization of cytochrome $c$ was hardly detected in normal U87 cells and pcDNA3.1 transfectants but flourished in the pcDNA3.1-Bec transfectants (Fig. 3D and E).

The activation of caspases, especially caspase-3/-9, is critical for the apoptotic pathway, as their activation leads to the release of cytochrome $c$ from the mitochondria and activation of the death signal $(25,26)$. We also measured caspase- 8 , caspase- 9 and caspase-3/-7 activity in different transfectants and untreated cells. As shown in Fig. 3D, no difference in the increase of caspase- 8 activity was observed between the pcDNA3.1-Bec transfectants and other transfectants. However, not only was there an increase of caspase-3/-7 activity but there was also an increase in caspase- 9 activity that was greater in the pcDNA3.1-Bec transfectants than in the other transfectants. These results indicated that Beclin 1 enhanced apoptosis in pcDNA3.1-Bec transfectants by enhancing caspase-3/-9 activity.

Overexpressed Beclin 1 can be combined with higher amounts of Bcl-2. We first examined the influence of altered expression of Beclin 1 on the expression of Bcl-2, Bcl-xl and Bax. As shown in Fig. 4A, the expression of the apoptosis-related proteins was not affected in pSUPER-Bec or pcDNA3.1Bec cells compared with controls. It can be inferred that the overexpression of Beclin 1 increased apoptosis since Beclin 1 sequestrated Bcl-2. Overexpressed Beclin 1 binds larger amounts of Bcl-2, which inhibited the pro-survival function of Bcl-2. Furthermore, apoptosis may be induced by the loss of Bcl-2 and its pro-survival functioning.

Immunoprecipitation (IP) was used to detect the binding of Bcl-2 to Beclin 1. We examined whether Beclin 1 protein expressed in the Beclin 1 gene transfectants bound to Bcl-2 family proteins. In pcDNA3.1-Bec transfectants, Beclin 1 was detected in the immunoprecipitates prepared with either the anti-Bcl-2 antibody or the anti-Bcl-xL antibody by IP (Fig. 4B). $\mathrm{Bcl}-2$ and $\mathrm{Bcl}-\mathrm{xL}$ were detected in immunoprecipitates

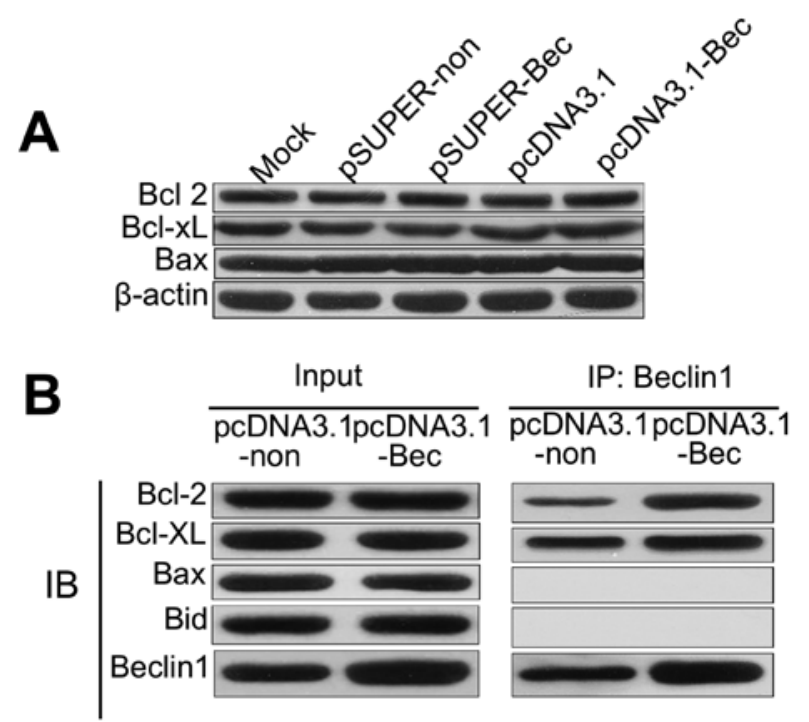

Figure 4. (A) The influence of altered expression of Beclin 1 on the expression of Bcl-2, Bcl-xl and Bax by transfection for $48 \mathrm{~h}$. The expression of the apoptosis-related proteins tested was not affected in pSUPER-Bec or pcDNA3.1-Bec cells compared with controls. $\mathrm{P}<0.05$. (B) In pcDNA3.1-Bec transfectants, Beclin 1 was detected in immunoprecipitates prepared with anti-Bcl-2 antibody or anti-Bcl-xL antibody by immunoprecipitation. Bcl-2 and Bcl-xL were detected in immunoprecipitates prepared with anti-Beclin 1 antibody. In addition, neither the Beclin 1-Bax complex nor the Beclin 1-Bid complex was detected. Nevertheless, the complex of Beclin 1 and Bcl-2 family proteins was hardly detected in the pcDNA3.1 transfectants. Overexpressed Beclin 1 cell lines can be combined with more Bcl2.

prepared with the anti-Beclin 1 antibody (Fig. 4B). In addition, neither the Beclin 1-Bax complex nor the Beclin 1-Bak complex was detected (Fig. 4B). However, a complex of Beclin 1 and the Bcl-2 family proteins was hardly detected in pcDNA3.1 transfectants (Fig. 4B). Overexpressed Beclin 1 cell lines can combine with more Bcl-2, which blocks Bcl-2 cells stimulated cells for survival.

\section{Discussion}

The tumor-suppression activity of Beclin 1 has been shown in several solid tumors including breast (4) and cervical cancer (15). Furthermore, Beclin 1 displays growth-inhibitory activity, regulation of autophagic cell death and induction of apoptosis. We found that enforced expression of Beclin 1 not only promoted autophagy in U87 cells but also induced apoptosis via binding to anti-apoptotic Bcl-2 family proteins such as Bcl-2 and Bcl-xL.

The interaction between Beclin 1 and its binding partners regulates the initial steps of autophagy (27). The association of Beclin 1 with hVps 34 and PI3k is essential for the induction of autophagy, and the autophagy-inducing activity of Beclin 1 is inhibited by the overexpression of Bcl-2 family members including Bcl-2, Bcl-xL and Mcl-1 $(28,29)$. There is evidence that the orthologs of Beclin 1, hVps34 and Bcl-2 do not form a trimolecular complex (30), suggesting that Beclin 1 can be present in only two different complexes, one that stimulates autophagy and involves an interaction with hVps34 and another that inhibits autophagy and involves an interaction with Bcl-2. Although it is clear that overexpression of $\mathrm{Bcl}-2$ can inhibit Beclin 1-mediated autophagy, it remains unclear whether the 
autophagy-inhibitory Beclin 1-Bcl-2 or Beclin 1-Bcl-xL interactions play any role in apoptosis.

Overexpression of Beclin 1 in U87 cells showed a reduction of cellular proliferation but also an augmentation of apoptosis. Therefore, apoptosis was crucial for cell death and proliferation in our experiments. We also demonstrated that Beclin 1 induced apoptosis in U87 cells by binding to Bcl-2 and Bcl-xL, leading to the release of cytochrome $c$ into the cytosol and subsequently activating caspases-3/-9. This result is in accordance with a study by Furuya et al (8). The interaction between Beclin 1 and the Bcl-2 family was first proposed following the observation that Beclin 1 binds to Bcl-2 and Bcl-xL but not to Bax.

Moreover, this model was proposed after observing that the interplay between Beclin 1 and $\mathrm{Bcl}-2$ family members indicated that Beclin 1 binds to $\mathrm{Bcl}-2$ and $\mathrm{Bcl}-\mathrm{xL}$ instead of Bax (31). Analyses of the Bcl-xL-binding domain of Beclin 1 support the postulate that Beclin 1 is a new member of the BH3-only family proteins. The finding that Beclin 1 is a newly discovered $\mathrm{BH} 3$-only protein immediately raises the possibility that Beclin 1 might have a proapoptotic role (32). BH3-only proteins can induce apoptosis by directly or indirectly stimulating the mitochondrion-permeabilizing activity of the pro-apoptotic multidomain proteins from the Bcl-2 family such as Bax and Bak (33). The formation of the Beclin 1-Bcl-2 or the Beclin 1-Bcl-xL complex may release Bax and Bak, which trigger mitochondrial membrane disruption and release of apoptogenic factors, such as cytochrome $c$ (24). It has been shown that in cells triggered to undergo apoptosis, mitochondrial cytochrome $c$ is released. Under these conditions and in the presence of dATP and Apaf-1, caspase-9 is activated, which in turn activates other caspases, such as caspase-3 $(34,35)$.

Some authors have suggested that Beclin 1 is not involved in apoptotic cell death. In previous studies, Yue et al (5) found no difference in apoptosis induced by ultraviolet light between wild-type mouse embryonic stem (ES) cells and Beclin 1-deficient ES cells. In a second set of studies, $\mathrm{Qu}$ et al (9) reported that the proportion of apoptotic cells in developing mammary glands of Beclin 1+/- mice did not differ from that in Beclin 1+/+ mice; it is worth noting that Bcl-2 and $\mathrm{Bcl}-\mathrm{xL}$ are expressed less in normal cells, including $\mathrm{ES}$ cells, than in cancer cells $(22,36,37)$. In the present study, we found that U87 cells showed less expression of Beclin 1 but more expression of the Bcl-2 family members than normal astrocytes. Therefore, these authors may have failed to find the pro-apoptotic function of Beclin 1, which involves inhibition of the anti-apoptotic function of Bcl-2 and Bcl-xL in nontransformed cells expressing low levels of these proteins (8).

Previous studies have shown that binding of $\mathrm{Bcl}-2$ to Beclin 1 inhibits Beclin 1-mediated autophagy (28). Thus, it is possible that the interaction between Beclin 1 and $\mathrm{Bcl}-2$ has dual effects: one is to modulate the apoptotic pathway and the second is to inhibit autophagy. These two effects may be mutually exclusive or may act together. Accordingly, upon complexation, Bcl-2 might inhibit Beclin 1's ability to participate in the autophagic process, whereas Bcl-2 will be concomitantly neutralized, thereby sensitizing the cells to apoptosis. In this way, the interaction between Beclin 1 and anti-apoptotic Bcl-2 family members may affect cell fate (11).
These observations indicate that Beclin 1, in addition to its role in autophagy, may play a role in apoptosis by neutralizing the anti-apoptotic proteins in the Bcl-2 family.

In mammalian cells, knockdown of Beclin 1 sensitized cells to apoptosis induction by starvation (38). Nevertheless, Beclin 1 downregulation also inhibited cell death induction by conditions in which essential pro-apoptotic mitochondrial outer membrane permeabilization (MOMP) or caspase activation was blocked $(39,40)$, and restoration of normal Beclin 1 levels in Beclin 1-deficient tumor cells facilitated the induction of cell death by vitamin D analogues (41). In these studies, the Beclin 1-mediated autophagy played key roles in cellular survival or death. However, in the present study, without metabolic stress or drugs, the levels of autophagic capacity were relatively low. When U87 cells were cultured in full nutrient and oxygen-rich conditions, the protective effect of energyprovision and nutrient-supply from autophagy was useless. Reduction of Beclin 1 levels by siRNA resulted in defective autophagy, but pSUPER-Bec transfectants had no significant difference from pSUPER-non transfectants in apoptosis and proliferation. We suggest that the low level of autophagic capacity in our experiment had little influence on cell proliferation.

Cancer often expresses high levels of Bcl-2-like antiapoptotic proteins to evade the apoptotic fate imposed by unscheduled cell proliferation, activation of oncogenes or DNA damage $(42,43)$. The downregulation of Beclin 1 in cancer $(4,44,45)$ may aggravate the anti-apoptotic effect of Bcl-2-like proteins.

In conclusion, our findings indicate that Beclin 1 not only enhanced autophagy and promoted apoptosis but also reduced cell proliferation. The above results suggested that the autophagy gene Beclin 1 plays an important role in fine tuning autophagy and apoptosis through interactions with Bcl-2 family members. These proteins may be suitable molecular targets for cancer treatment.

\section{Acknowledgements}

The authors thank Dr Chongli Yan and Dr Weiwei Guo for their assistance and critical appraisal of the manuscript. The present study was supported by grants from the China Postdoctoral Science Foundation (no. 20100480568).

\section{References}

1. Klionsky DJ, Cregg JM, Dunn WA Jr, et al: A unified nomenclature for yeast autophagy-related genes. Dev Cell 5: 539-545, 2003.

2. Levine B and Yuan J: Autophagy in cell death: an innocent convict? J Clin Invest 115: 2679-2688, 2005.

3. Clarke PG: Developmental cell death: morphological diversity and multiple mechanisms. Anat Embryol (Berl) 181: 195-213, 1990.

4. Liang XH, Jackson S, Seaman M, et al: Induction of autophagy and inhibition of tumorigenesis by beclin 1 . Nature 402: 672-676, 1999.

5. Yue Z, Jin S, Yang C, Levine AJ and Heintz N: Beclin 1, an autophagy gene essential for early embryonic development, is a haploinsufficient tumor suppressor. Proc Natl Acad Sci USA 100: 15077-15082, 2003.

6. Zeng X, Overmeyer JH and Maltese WA: Functional specificity of the mammalian Beclin-Vps34 PI 3-kinase complex in macroautophagy versus endocytosis and lysosomal enzyme trafficking. J Cell Sci 119: 259-270, 2006. 
7. Kang R, Zeh HJ, Lotze MT and Tang D: The Beclin 1 network regulates autophagy and apoptosis. Cell Death Differ 18: 571-580, 2011.

8. Furuya D, Tsuji N, Yagihashi A and Watanabe N: Beclin 1 augmented cis-diamminedichloroplatinum induced apoptosis via enhancing caspase-9 activity. Exp Cell Res 307: 26-40, 2005

9. Qu X, Yu J, Bhagat G, et al: Promotion of tumorigenesis by heterozygous disruption of the beclin 1 autophagy gene. J Clin Invest 112: 1809-1820, 2003

10. Adams JM and Cory S: The Bcl-2 protein family: arbiters of cell survival. Science 281: 1322-1326, 1998.

11. Erlich S, Mizrachy L, Segev O, et al: Differential interactions between Beclin 1 and Bcl-2 family members. Autophagy 3 : 561-568, 2007.

12. Trisciuoglio D, De Luca T, Desideri M, et al: Removal of the BH4 domain from Bcl-2 protein triggers an autophagic process that impairs tumor growth. Neoplasia 15: 315-327, 2013.

13. Oh S, Xiaofei E, Ni D, et al: Downregulation of autophagy by Bcl-2 promotes MCF7 breast cancer cell growth independent of its inhibition of apoptosis. Cell Death Differ 18: 452-464, 2011

14. Ricci A, Cherubini E, Scozzi D, et al: Decreased expression of autophagic beclin 1 protein in idiopathic pulmonary fibrosis fibroblasts. J Cell Physiol 28: 1516-1524, 2012.

15. Wang ZH, Xu L, Duan ZL, Zeng LQ, Yan NH and Peng ZL: Beclin 1-mediated macroautophagy involves regulation of caspase-9 expression in cervical cancer HeLa cells. Gynecol Oncol 107: 107-113, 2007.

16. Trincheri NF, Follo C, Nicotra G, Peracchio C, Castino R and Isidoro C: Resveratrol-induced apoptosis depends on the lipid kinase activity of Vps34 and on the formation of autophagolysosomes. Carcinogenesis 29: 381-389, 2008.

17. Wen PY and Kesari S: Malignant gliomas in adults. N Engl J Med 359: 492-507, 2008.

18. Furnari FB, Fenton T, Bachoo RM, et al: Malignant astrocytic glioma: genetics, biology, and paths to treatment. Genes Dev 21: 2683-2710, 2007.

19. Miracco C, Cosci E, Oliveri G, et al: Protein and mRNA expression of autophagy gene Beclin 1 in human brain tumours. Int J Oncol 30: 429-436, 2007.

20. Huang X, Bai HM, Chen L, Li B and Lu YC: Reduced expression of LC3B-II and Beclin 1 in glioblastoma multiforme indicates a down-regulated autophagic capacity that relates to the progression of astrocytic tumors. J Clin Neurosci 17: 1515-1519, 2010

21. Brummelkamp TR, Bernards R and Agami R: A system for stable expression of short interfering RNAs in mammalian cells. Science 296: 550-553, 2002.

22. Rieger L, Weller M, Bornemann A, Schabet M, Dichgans J and Meyermann R: BCL-2 family protein expression in human malignant glioma: a clinical-pathological correlative study. J Neurol Sci 155: 68-75, 1998.

23. Klionsky DJ, Abeliovich H, Agostinis P, et al: Guidelines for the use and interpretation of assays for monitoring autophagy in higher eukaryotes. Autophagy 4: 151-175, 2008.

24. Gross A, McDonnell JM and Korsmeyer SJ: BCL-2 family members and the mitochondria in apoptosis. Genes Dev 13: 1899-1911, 1999

25. Hausmann G, O'Reilly LA, van Driel R, et al: Pro-apoptotic apoptosis protease-activating factor 1 (Apaf-1) has a cytoplasmic localization distinct from Bcl-2 or Bcl- $\mathrm{x}_{\mathrm{L}}$. J Cell Biol 149 : 623-634, 2000

26. Cory S, Huang DC and Adams JM: The Bcl-2 family: roles in cell survival and oncogenesis. Oncogene 22: 8590-8607, 2003.
27. Shintani T and Klionsky DJ: Autophagy in health and disease: a double-edged sword. Science 306: 990-995, 2004.

28. Pattingre S, Tassa A, Qu X, et al: Bcl-2 antiapoptotic proteins inhibit Beclin 1-dependent autophagy. Cell 122: 927-939, 2005.

29. Maiuri MC, Le Toumelin G, Criollo A, et al: Functional and physical interaction between $\mathrm{Bcl}-\mathrm{X}_{\mathrm{L}}$ and a $\mathrm{BH} 3$-like domain in Beclin-1. EMBO J 26: 2527-2539, 2007.

30. Takacs-Vellai K, Vellai T, Puoti A, et al: Inactivation of the autophagy gene bec-1 triggers apoptotic cell death in C.elegans. Curr Biol 15: 1513-1517, 2005.

31. Liang XH, Kleeman LK, Jiang HH, et al: Protection against fatal Sindbis virus encephalitis by beclin, a novel Bcl-2-interacting protein. J Virol 72: 8586-8596, 1998.

32. Feng W, Huang S, Wu H and Zhang M: Molecular basis of Bcl-xL's target recognition versatility revealed by the structure of Bcl-xL in complex with the BH3 domain of Beclin-1. J Mol Biol 372: 223-235, 2007.

33. Kroemer G, Galluzzi L and Brenner C: Mitochondrial membrane permeabilization in cell death. Physiol Rev 87: 99-163, 2007.

34. Zou H, Henzel WJ, Liu X, Lutschg A and Wang X: Apaf-1, a human protein homologous to $C$. elegans CED-4, participates in cytochrome c-dependent activation of caspase-3. Cell 90: 405-413, 1997.

35. Li P, Nijhawan D, Budihardjo I, et al: Cytochrome c and dATPdependent formation of Apaf-1/caspase-9 complex initiates an apoptotic protease cascade. Cell 91: 479-489, 1997.

36. Okazawa H, Shimizu J, Kamei M, Imafuku I, Hamada H and Kanazawa I: Bcl-2 inhibits retinoic acid-induced apoptosis during the neural differentiation of embryonal stem cells. J Cell Biol 132: 955-968, 1996.

37. Orelio C, Harvey KN, Miles C, Oostendorp RA, van der Horn K and Dzierzak E: The role of apoptosis in the development of AGM hematopoietic stem cells revealed by Bcl-2 overexpression. Blood 103: 4084-4092, 2004

38. Lum JJ, Bauer DE, Kong M, et al: Growth factor regulation of autophagy and cell survival in the absence of apoptosis. Cell 120: 237-248, 2005

39. Shimizu S, Kanaseki T, Mizushima N, et al: Role of Bcl-2 family proteins in a non-apoptotic programmed cell death dependent on autophagy genes. Nat Cell Biol 6: 1221-1228, 2004.

40. Yu L, Alva A, Su H, et al: Regulation of an ATG7-beclin 1 program of autophagic cell death by caspase-8. Science 304 : $1500-1502,2004$.

41. Hoyer-Hansen M, Bastholm L, Mathiasen IS, Elling F and Jaattela M: Vitamin D analog EB1089 triggers dramatic lysosomal changes and Beclin 1-mediated autophagic cell death. Cell Death Differ 12: 1297-1309, 2005.

42. Amundson SA, Myers TG, Scudiero D, Kitada S, Reed JC and Fornace AJ Jr: An informatics approach identifying markers of chemosensitivity in human cancer cell lines. Cancer Res 60: 6101-6110, 2000.

43. Kirkin V, Joos S and Zornig M: The role of Bcl-2 family members in tumorigenesis. Biochim Biophys Acta 1644: 229-249, 2004.

44. Paglin S, Hollister T, Delohery T, et al: A novel response of cancer cells to radiation involves autophagy and formation of acidic vesicles. Cancer Res 61: 439-444, 2001.

45. Shen Y, Li DD, Wang LL, Deng R and Zhu XF: Decreased expression of autophagy-related proteins in malignant epithelial ovarian cancer. Autophagy 4: 1067-1068, 2008. 